\title{
Unhealthy Communication: Health Care Communication Between Majority and Minority Cultural Groups
}

\section{Adiki Puplampu}

\begin{abstract}
Effective communication is important in almost every aspect of life. Within the medical setting, effective communication is paramount because the consequences of miscommunication can be deadly. The purpose of this paper is to examine health care communication through the theoretical lens of anxiety-uncertainty management theory (A-UM) and explore how it is affected by differential power dynamics. Drawing on the literature, it will look at the communication between doctors from majority groups and patients from minority groups in order to advance the following argument: the communicative relationship between doctors from dominant groups and patients from minority groups is negatively affected by A-UM theory as influenced by the asymmetrical power relationship between the two parties. This relationship is problematic because communication within a medical context is important for patient satisfaction, recall of information, and outcomes.
\end{abstract}

\section{Introduction}

The doctor-patient relationship has existed for thousands of years, with the word "doctor" first appearing in the English language around the 14th century (Merriam-Webster, n.d). Interpersonal interactions between doctors and patients are crucial because they are the primary method of information exchange between the two parties. Even with all the technology at our disposal, the diagnostic work of doctors is still most effective when it is done face-to-face with patients (Ong et al., 1995, p. 903). Consequently, the importance of competent communication in these interactions cannot be overstated.

In today's world, where cultural diversity is commonplace, there still exist majority and minority cultural groups within most nations. As a result of their group membership, these groups' lived experiences are different, and these differences can affect their communicative experiences. In the medical context where communicative breakdowns can have serious consequences, what is the communication like between members of majority and minority groups in the medical setting? This paper argues that the asymmetrical power relationship triggers or influences anxiety-uncertainty management theory's (A-UM) effects in the communicative relationship between doctors from majority cultures and patients from minority cultures, which is problematic because communication within a medical context is important for patient satisfaction, recall of information, and outcomes. To establish this argument the paper explores how communication as influenced by A-UM theory between these two groups can affect the aforementioned patient variables, ultimately finding that patient outcomes are influenced by the patient-practitioner relationship and that patient satisfaction is lowered when 


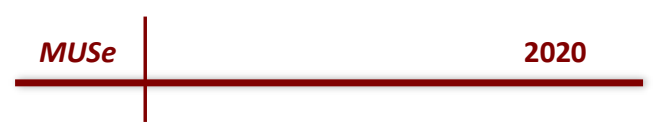

patients feel that they have not been provided with enough information, and are unable to recall information.

Following the introduction, the literature review explores some of the trends in medical communication literature, which include discussion about the variables that affect doctor-patient communication and solutions to poor intercultural medical communication. The section after the literature review establishes that power disparities exist between doctors and patients and between majority and minority cultural groups. The next section discusses the negative consequences of poor intercultural communication between patients and doctors regarding patient satisfaction, information recall, and outcomes before concluding that without clear expressions of their communicative differences, the communication between these two groups will continue to be compromised.

\section{Literature Review}

Literature specifically on communication between minority and majority cultural groups in health care is limited. But significant research has been done on general aspects of doctor-patient communication and intercultural communication in health care. Ong et al. (1995) provide a baseline of metrics from which to view doctor-patient communication. They identify "ereating [sic] a good inter-personal relationship, exchanging information and making treatment-related decisions" as the three main functions of doctor-patient communication (pp. 903-904). Similarly Schinkel et al. (2016) identify satisfaction, fulfillment of information needs, and understanding of information as the main outcomes of communicative interactions in medical settings (p. 1254).

With these variables identified, literature concentrated on doctor-patient communication looks at the communicative behaviours of both parties. Ong et al. (1995) discuss the difference between instrumental or task-focused communication and affective socio-emotional behaviour (p. 906). Instrumental behaviour is focused on the information exchange aspect of the interaction; examples of instrumental communication include "giving information, asking questions, [and] counselling" (p. 906). On the other hand, affective communication is more concerned with relationship building between doctors and patients (p. 906). It includes behaviours like "showing concern, giving reassurance, [and] showing approval" (p. 906). Street (1991) also discusses communication styles, focusing on how patient communication styles affect information exchange. After collecting data on doctor's appointments held during a sixweek span at a hospital in the southwestern United States, Street found that "through their communicative styles, patients can exert considerable control of the amount of information they receive from doctors" (p. 546). The research found that patients' most effective communication tool is asking questions (p. 546).

Research looking at intercultural medical communication takes a critical approach and also explores solutions to intercultural communication barriers. A-UM theory provides a theoretical explanation for some of the barriers to intercultural communication. Popularized by Gudykunst, A-UM theory asserts that "managing uncertainty and anxiety are central processes that influence the effectiveness of our communication with others in interpersonal and group encounters" (Duronto et al., 2005, p. 550). One of the main assumptions of the theory is that when individuals experience high levels of anxiety in a communication situation their information processing becomes lower level and they communicate less effectively (Gudykunst \& Nishida, 


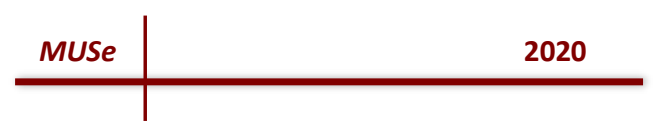

2001 , p. 56). And when their feelings of uncertainty are high, their confidence is affected, which impedes their ability to "predict or explain others' attitudes, feelings, or behaviors" (p. 56). The theory is strongly tied to intercultural communication because typically one, if not both parties, experience heightened anxiety in an intercultural communication scenario out of fear of miscommunication. Presbitero \& Attar (2018) posit that this anxiety "and worry when communication cross-culturally [could] relate to low effectiveness when sharing information with others" (p. 37). Their study surveyed Australian employees on their perceptions around intercultural communication. The survey found that cultural intelligence (CQ) was an important factor in reducing anxiety and creating more optimal knowledge-sharing conditions between intercultural participants.

Likewise, Gibson and Zhong (2005) conducted a survey with medical providers and their patients in the United States. The survey asked the medical providers to reflect on their intercultural communication abilities and the patients to provide feedback on the intercultural communication competence (ICCC) of the medical providers (p. 621). They found a positive relationship between ICCC and empathy based on the opinions expressed by both the medical staff and patients (p. 629). The research did not find a strong connection between bilingualism and ICCC but did find that if medical providers had intercultural experience, their ICCC skills were stronger (p. 630). Ulrey and Amason (2001) have a similar focus as Gibson and Zhong with their hypothesis that "culturally sensitive health care providers are indeed better communicators" (p. 454). They also administered a survey that measured cultural sensitivity, effective intercultural communication, and anxiety (pp. 454-455). Similar to Gibson and Zhong's findings regarding empathy, Ulrey and Amason discovered that there was a positive relationship between cultural sensitivity and ICCC (p. 458). Research on intercultural communication is in agreement that developing ICCC or CQ is important in improving communication between individuals of different cultures. This paper will be combining the discussions around doctorpatient communication and intercultural communication as it relates to A-UM theory in order to examine what creates breakdowns in intercultural doctor-patient communication.

\section{Discussion}

Every interpersonal interaction or relationship is, in some way, affected by power dynamics (Nimmon \& Stenfors-Hayes, 2016, p. 2). In the case of the doctor-patient interaction, the power relationship is one of imbalance. In face-to-face interactions with patients, the "unequal relationship is a product of physicians possessing legitimized, referent, and expert power and patients being reliant on physicians to provide the care and services they need" (Nimmon \& Stenfors-Hayes, 2016, p. 2). As a result, doctors have the ability to control the nature of the interaction without input from patients, and for many years they did so. Before the medical community shifted to a more "shared decision-making approach," the relationship between doctors and patients was defined by its paternalistic nature (Ong et al.,1995, p. 905). When the shift occurred, doctors recognized that in order to make decisions about their health, patients need to have all the relevant information (Ong et al., 1995, p. 905). Regardless of this shift, the relationship between patients and doctors is still affected by patients' reliance on doctors' expertise for their wellbeing. The addition of cultural barriers to the already delicate relationship 


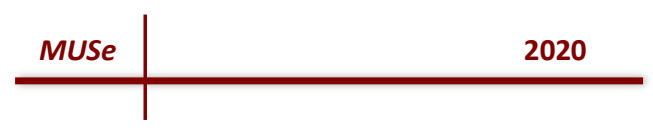

between doctors and patients complicates matters further. In Canada, the increasingly diverse population makes this a pertinent issue.

In 2017, Canada became home to over 286,000 new permanent residents (Government of Canada, 2019, para. 3). And the Canadian government has plans to steadily increase the number of immigrants welcomed into Canada to 340,000 by this year (Government of Canada, 2019, para. 4). The growing inflow of immigrants contributes to the already diverse Canadian population. In fact, in 2016, the number of cultural backgrounds represented in Canada was over 250, and these populations have likely grown in subsequent years (Vézina et al., 2017, para. 1). With all of these new cultures comes increased intercultural communication. In the medical setting, this intercultural communication is affected not only by the inherent power imbalance between patients and doctors but also by the power imbalance between majority and minority cultural groups.

Fairhurst and Snavely (1983), while discussing tokenism, succinctly express the reality of being a minority: "Deviation from the majority confers a lower status on tokens, and because status is usually correlated with power, tokens have less power" (p. 293). While the term token is used in this quote, the sentiment still reflects the general relationship between cultural majorities and minorities. Cultural minorities living in majority cultures have to coordinate the reality of their former culture with their new one, and despite their efforts, members of the majority culture still identify them as minorities.

Delving into the intricacies of the general power dynamics between majority and minority cultural groups is beyond the scope of this study. As such, the main takeaway for the purposes of this paper is that these dynamics exist. We have already established that there are numerous cultural groups represented in Canada alone. To build the argument that the power distance between doctors and patients is heightened when doctors come from majority groups and patients come from minority groups, which in turn creates anxiety in accordance with A-UM theory, we first need to establish that there are, in fact, majority group practitioners. According to 2016 research from the American Medical Association, 29.1\% of doctors are foreign-born (Santhanam, 2018, para. 5). Based on these numbers, we can estimate that approximately $70 \%$ of doctors are native-born and, therefore, part of the majority cultural group. Unfortunately, I was unable to find data for Canadian doctors, but this American data provides a basis for this paper's analysis. While some might argue that this percentage is simply a reflection of population demographics, I would contend that distribution is not the issue. The issue is how these doctors communicate with their patients from minority cultural backgrounds. One final detail to note is that these numbers do not reflect ethnic minorities who may have been born in the United States but raised with their cultural backgrounds.

Two interrelated processes of power have now been established: an asymmetric power dynamic exists in the doctor-patient relationship, and there is a power dynamic between minority and majority cultural groups. It has also been determined, by looking at the figures on immigrant doctors, that a large number of physicians are likely from majority cultural backgrounds. Now that this framework has been established, we will discuss the impact of these power differentials in medical communication. While there are several outcomes of doctor-patient communication, this paper will focus specifically on the impact of intercultural communication on patient satisfaction, recall of information, and outcomes. 


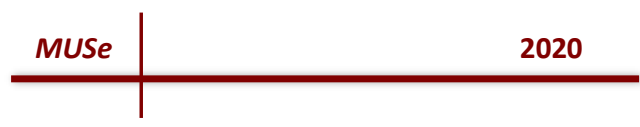

According to Ong et al. (1995), one important outcome of doctor-patient communication is overall patient satisfaction (p. 910). The objectives behind a doctor's appointment are highly situational, but at some point in almost every interaction with a doctor, some form of information exchange is required on either the part of the doctor or on the part of the patient. In their literature review, Ong et al. found that patient satisfaction increased when patients felt that they were being provided with enough information (p. 910). If patients from minority cultures experience anxiety as a result of their medical issue or the multiple power imbalances at play in the interaction, based on A-UM theory, they might find it hard to ask for further information. If they fail to receive the information they came for, it is likely that their overall satisfaction with the interaction will be reduced.

A-UM theory contends that a " high presence of uneasiness and worry when communicating cross-culturally... relate(s) to low effectiveness when sharing information with others," and this lack of information sharing can affect patient satisfaction (Presbitero \& Attar, 2018, p. 37). A significant portion of the doctor-patient relationship is centered around information sharing. Without clear lines of communication for that information to be shared, the relationship is compromised and the effectiveness of the doctor as a medical practitioner is jeopardized. Intercultural communication is also compromised in the medical setting by Schinkel et al.'s (2016) finding that "ethnic minority patients are less participative in medical consultations compared to ethnic majority patients" (p. 1251). If they are less participative, then they are less likely to voice any concerns they have or ask for more information than the doctor provides. In this way, the cultural and power barriers between them and the doctor affect their medical experience.

In medical appointments, doctors are often communicating vital and sometimes complex information to patients. After the appointment, it is important that patients remember what they have been told so that they can apply it or do further research as necessary. In the literature, Schinkel et al. (2016) found that minority patients end up with less comprehension of the information they receive compared to patients from majority populations (p. 1251). This may be a consequence of doctors' approaches to information giving. Street (1991) posits that doctor's attitudes toward providing information "are related to physicians' attitudes towards patients" ( $p$. 541). As mentioned in the previous paragraph, the literature has found that, generally, patients from minority cultural backgrounds engage less in medical contexts (p. 1251). According to Schinkel et al., minority patients "ask fewer questions and make fewer requests and treatment suggestions" (p. 1251). If doctors develop a negative view of a minority patient because of this lack of engagement, or if they just believe that they won't appreciate detailed information, they might not provide it. In this way, culture has become a barrier in the communication between doctors and patients.

While the barriers to communication theorized by A-UM theory do play a part in these barriers, minority patients' reluctance to engage in their medical appointments might also be a consequence of their cultural upbringing. For example, Geist-Martin (2015) profiles a case study in which a Chinese woman, after having a heart attack, was asked to come back to the doctor's office after two weeks for a follow-up visit (p. 324). The case study explains the woman's failure to show up, saying, "it is likely that she never intended to do so but agreed because he [the doctor] was an authority figure" (p. 324). While the outcome of this case might just be a cultural coincidence, it still demonstrates the larger argument that the asymmetrical power relationship 


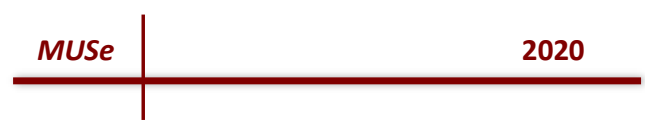

affected the communication. And this power relationship, in turn, created apprehension or nervousness in the patient in relation to A-UM theory that affected her communication. The miscommunication occurred because the woman respected the doctor as a person of authority, and as a result, did not clearly express her intentions. The final function of health care communication that will be discussed in this paper, and arguably the most important one, is patient outcomes.

Of course, "the ultimate aim of medical care is to produce optimal health outcomes" (Ong et al., 1995, p. 912). Even though health outcomes are affected by multiple variables, some of which are out of even the doctor's control, research has found "that the physicianpatient relationship may have important consequences for patients' health outcomes" (p. 912). Researchers have found that the relationship between doctors and patients becomes a "form of social support" which helps to "reinforce patient's self-confidence, motivation, and positive view of their health status" (Kaplan et al., as cited in Ong et al, 1995, p. 912). This research confirms that the communicative relationship between patients and doctors has the potential to affect their health outcomes. If patients feel dissatisfied with the interactions they have with their doctors or are unable to recall information from the interaction, intercultural communication has failed, and the doctor-patient relationship is not performing its role as "social support." This is especially troubling when you consider that majority groups do not face this added cultural communication and power difference barrier. Without these barriers, their communicative interactions with their doctors are conceivably different than those of minority cultural patients. One final thing to note is that while breakdowns in intercultural communication are frustrating for patients, they are also distressing to the health care providers themselves (Gibson \& Zhong, 2005, p. 622). People who spend decades in school likely want to apply their skills as effectively as possible. As much as patients want to communicate effectively, doctors want to do so just as much.

\section{Conclusion}

Effective communication within a medical context is critical for patient satisfaction, information recall, and health outcomes. These outcomes are affected when the asymmetrical power relationship between doctors from majority cultural backgrounds and patients from minority cultural backgrounds becomes a communication barrier. As Gibson and Zhong (2005) put it, "Communicative misunderstandings between patient and provider can lead to simple dissatisfaction, misdiagnosis, lack of medical care, or even death" (p. 622). When it comes to patient satisfaction and information recall, research has found that patients from minority groups engage less than members of majority groups in medical settings (Schinkel et al., 2016, p. 1251). This paper argued that this decreased participation, while potentially influenced by cultural values, is actually a consequence of the intimidation that patients experience as a result of the power differential between themselves and the doctor. This intimidation plays into A-UM theory, which theorizes that the process of managing anxiety caused by communication situations affects the efficacy of the communication (Gudykunst \& Nishida, 2001). In the medical context, this can impact communication and information sharing between doctors and patients. Research has also found that patient satisfaction is contingent upon patient perception that they have been provided all relevant information (Ong et al., 1995, p. 910). Because minority patients 
are less likely to actively participate in medical appointments, they will receive less information and therefore be less satisfied. With less information, they will have less to recall. The failure of these two medical communication functions also likely influences patient outcomes, as research has found that doctor-patient communication does have an impact on patient outcomes (Ong et al., 1995, p. 912). Further research into this topic could look specifically at the communicative experiences of specific minority cultural groups, as well as the role language barriers play.

Doctors, in their role as knowledge practitioners, can ease the anxiety and uncertainty patients face in intercultural contexts by making an effort to put their patients at ease and by developing cultural intelligence (CQ) (Presbitero \& Attar, 2018). Developing CQ does not happen passively and can be approached "through experiential learning..., engaging in reflective observation and abstract conceptualization, and active experimentation" (p. 41). Through these $C Q$ practices, doctors can create a neutral communicative ground for patients to interact with them upon. " $C Q$ can be relevant in lessening the negative influence of both anxiety and uncertainty on intercultural communication effectiveness" (p. 37). And communication is a two-way street. As much as doctors need to adjust their communication styles to meet patients' needs, patients also have to be clear in the communication style that works best for them. Without this clear communication, patients and doctors allow their cultural status and asymmetrical power relationship to reduce the effectiveness of healthcare communication. 


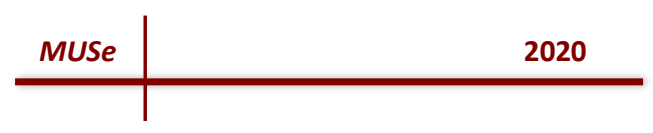

\section{References}

Duronto, P. M., Nishida, T., \& Nakayama, S. (2005). Uncertainty, anxiety, and avoidance in communication with strangers. International Journal of Intercultural Relations, 29(5), 549-560. https://doi.org/10.1016/i.ijintrel.2005.08.003

Fairhurst, G. T., \& Snavely, B. K. (1983). Majority and token minority group relationships: Power acquisition and communication. Academy of Management Review, 8(2), 292-300. https://doi.org/10.5465/AMR.1983.4284739

Geist-Martin, P. (2015). Negotiating cultural understanding in health care communication. In Samovar, L. A., Porter, R. E., McDaniel, E. R., \& Sexton Roy, C. (Eds.), Intercultural communication: A reader (14th ed., pp. 320-328). Boston, MA: Wadsworth, Cengage Learning.

Gibson, D., \& Zhong, M. (2005). Intercultural communication competence in the healthcare context. International Journal of Intercultural Relations, 29(5), 621-634. https://doi.org/10.1016/i.ijintrel.2005.07.008

Government of Canada. (2019). 2018 annual report to parliament on immigration. https://www.canada.ca/en/immigration-refugees-citizenship/corporate/publicationsmanuals/annual-report-parliament-immigration-2018/report.html

Gudykunst, W. B., \& Nishida, T. (2001). Anxiety, uncertainty, and perceived effectiveness of communication across relationships and cultures. International Journal of Intercultural Relations, 25(1), 55-71. https://doi.org/10.1016/S0147-1767(00)00042-0

Merriam-webster. (n.d). The history of "doctor". https://www.merriam-webster.com/words-atplay/the-history-of-doctor

Nimmon, L., \& Stenfors-Hayes, T. (2016). The "handling" of power in the physician-patient encounter: Perceptions from experienced physicians. BMC Medical Education, 16, 1-9. https://doi.org/10.1186/s12909-016-0634-0

Ong, L. M. L., De Haes, J. C. J. M., Hoos, A. M., \& Lammes, F. B. (1995). Doctor-patient communication: A review of the literature. Social Science \& Medicine, 40(7), 903-918. https://doi.org/10.1016/0277-9536(94)00155-M

Presbitero, A., \& Attar, H. (2018). Intercultural communication effectiveness, cultural intelligence and knowledge sharing: Extending anxiety-uncertainty management theory. International Journal of Intercultural Relations, 67, 35-43. https://doi.org/10.1016/j.ijintrel.2018.08.004 
Santhanam, L. (2018). New study shows 1 in 6 U.S health care workers are immigrants. PBS News Hour. https://www.pbs.org/newshour/health/new-study-shows-1-in-6-u-s-healthcare-workers-are-immigrants

Schinkel, S., Schouten, B. C., Street, R. L., Jr., van den Putte, B., \& van Weert, J. C. M. (2016). Enhancing health communication outcomes among ethnic minority patients: The effects of the match between participation preferences and perceptions and doctor-patient concordance. Journal of Health Communication, 21(12), 1251-1259. https://doi.org/10.1080/10810730.2016.1240269

Street Jr., R. L. (1991). Information-giving in medical consultations: The influence of patients' communicative styles and personal characteristics. Social Science \& Medicine, 32(5), 541-548. https://doi.org/10.1016/0277-9536(91)90288-N

Ulrey, K. L., \& Amason, P. (2001). Intercultural communication between patients and health care providers: An exploration of intercultural communication effectiveness, cultural sensitivity, stress, and anxiety. Health Communication, 13(4), 449-463. https://doi.org/10.1207/S15327027HC1304 06

Vézina, M., Maheux, H., Houle, R., Corbeil, J.P. (2019). Ethnic and cultural origins of Canadians: Portrait of a rich heritage. https://www12.statcan.gc.ca/censusrecensement/2016/as-sa/98-200-x/2016016/98-200-x2016016-eng.cfm 